\title{
Nulling interferometry without achromatic phase shifters: latest results
}

\author{
Julien Spronck, Silvania F. Pereira and Joseph J. M. Braat \\ Optics Research Group, Faculty of Applied Sciences, \\ Delft University of Technology, \\ Lorentzweg 1, NL-2628 CJ Delft, The Netherlands
}

\begin{abstract}
We discuss the previously-reported measurements of a three-beam nulling interferometer without achromatic phase-shifters, using delay lines only. The theoretical rejection ratio of a few thousand has not been achieved experimentally. In order to explain the obtained results, some direct spectral and polarization measurements have been performed. We present here the latest results and discuss some asymmetries in the interference patterns.
\end{abstract}

Keywords: Interferometry, Astronomical optics, Nulling

\section{INTRODUCTION}

Search for exoplanets has become a very active field in the last ten years, since the discovery of the first exoplanet in 1995 by Mayor and Queloz. ${ }^{1}$ Since that moment, more than one hundred and fifty planets have been detected. Most of them were found by indirect methods, ${ }^{2,3}$ which means that only some effects that the planet has on its star have been detected.

Direct detection of Earth-like exoplanets is very challenging because of the huge brightness contrast between the star and the planet and because of their small angular separation. Nevertheless, this should be possible with a technique called nulling interferometry, ${ }^{4}$ which consists in looking at a star-planet system with an array of telescopes, and then combining the light from these telescopes in such a way that destructive interference occurs for the star light and constructive interference for the planet light. The ratio between the intensities corresponding to constructive and destructive interference is called the rejection ratio. To be able to detect an Earth-like planet, this ratio should be of the order of $10^{6}$ in the mid-infrared region.

This high rejection ratio should be achieved in a wide spectral band (typically from 5-18 $\mu \mathrm{m}$ ), in order to obtain spectral information from the planet. To cancel the light from the on-axis star in such a wide band, most nulling interferometers use an achromatic phase shifter. Such a device creates a phase shift quasi-independent on the wavelength and, therefore, allows a high rejection ratio in a broad band. But, technically, it is quite challenging to build these phase shifters.

However, it has been shown that high rejection ratio is possible with delay lines only as phase-shifters. ${ }^{5,6}$ In a wide spectral band, the chromatism induced by the delay lines would drastically limit the rejection ratio. This problem is overcome by increasing the number of beams. Indeed, in the family of interferometers proposed by Mieremet, ${ }^{7}$ a larger number of beams allows less stringent requirements in terms of achromaticity.

An experimental set-up has been built to demonstrate this principle. In this paper, we will show the latest results obtained with this set-up. In Section 2, we describe the experimental set-up. In Section 3, we show the measurements previously reported. In Sections 4 and 5, we present some spectral and polarization measurements to explain these results. The latest measurements are then discussed in Section 6 and our conclusions are summarized in Section 7. 


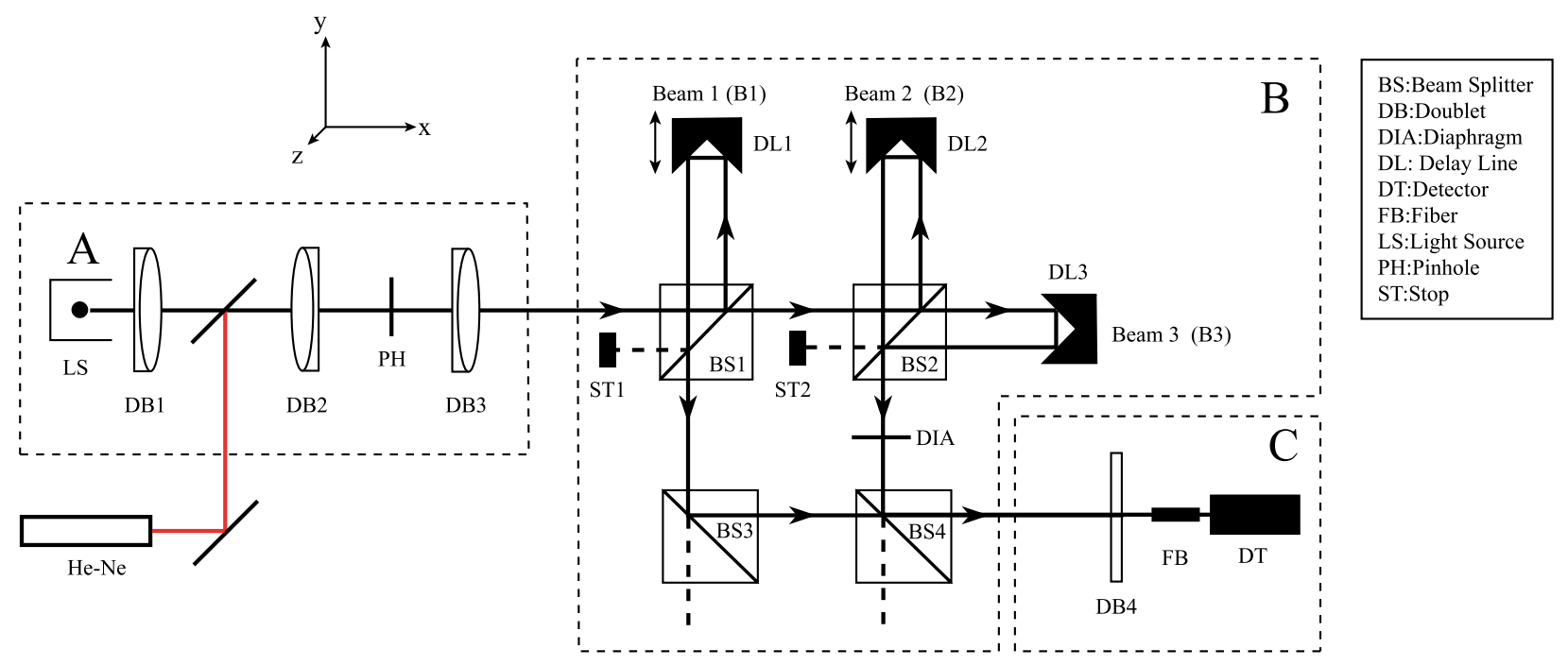

Figure 1. The experimental set-up can be divided in three blocks: the star simulator (A), the interferometer (B) and the detection stage $(\mathrm{C})$.

\section{EXPERIMENTAL SET-UP}

The experimental set-up, ${ }^{6}$ depicted in Fig. 1, is a three-beam nulling interferometer. It can be divided in three blocks: the star simulator, the interferometer and the detection stage.

In the star simulator, light from a Xe arc light source (LS) is focused onto a $5 \mu$ m-pinhole (PH), acting as a point source. Light is then collimated with a doublet (DB3) to form a 8-mm beam. With color filters, we chose the spectral band from 500 to $650 \mathrm{~nm}$. Furthermore, for alignment purpose, we use a He-Ne laser that we focus onto the same pinhole using a folding mirror.

The interferometer consists of four beam-splitters (BS) to create and recombine three beams. Each of these three beams goes, before recombination, to different retro-reflectors acting as delay lines. The relative optical path differences between the three beams can be varied by changing the position of the delay lines (DL) via piezo-actuators.

After recombination, the beams are sent to a single-mode optical fiber (FB) for modal filtering. ${ }^{8}$ The fiber is then connected to a powermeter (DT) to detect the resulting intensity.

The interference pattern is built by measuring the intensity as a function of the position of the delay lines. We define the rejection ratio as the ratio between the maximum and the minimum of the interference pattern.

\section{PREVIOUS RESULTS}

In this experimental set-up, a rejection ratio of $10^{6}$ is not possible due to the chromatism induced by the delay lines and the limited number of beams. With two beams, the theoretical rejection ratio that we could reach in such a wide spectral band is of the order of 100. The three-beam nulling interferometer would allow a rejection ratio of a few thousands with the appropriate amplitude distribution.

The optimal amplitude ratio is 1:2:1, which corresponds to a power ratio of 1:4:1. Reaching this amplitude distribution in an achromatic way without affecting the phase is not trivial. For that purpose, we use a diaphragm that will limit the size (and therefore the power) of two of the three beams. Note that this diaphragm can distort the spectral shape of the beams due to the chromatic coupling with the single-mode optical fiber. ${ }^{9}$

The goal of the experiment was to show that the optimal amplitude ratio would lead to a relatively high rejection ratio compared to the conventional 1:1:1 amplitude distribution. The theoretical and measured rejection

E-mail: J.F.P.Spronck@tudelft.nl 


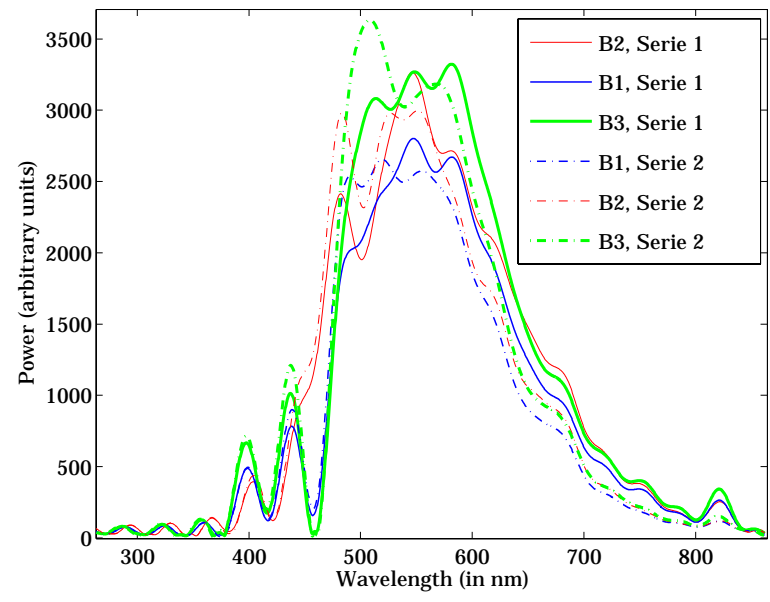

(a)

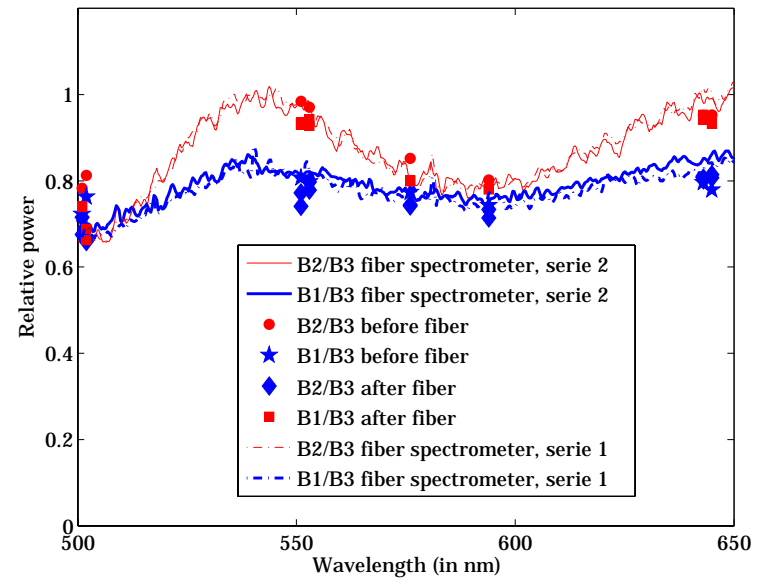

(b)

Figure 2. (a) Spectra of the three beams $(B 1, B 2, B 3)$ measured with a multi-mode fiber spectrometer. The spectra have been numerically filtered. (b) Relative spectral measurements with interference filters (individual marks) and a multi-mode fiber spectrometer (lines). For each method, several series of measurements have been performed.

ratios $^{6}$ are listed in Table (1). We can see that the equal-amplitude rejection ratio matches the expectations much better than in the case of the 1:2:1 amplitude distribution. In the following sections, we investigate the possible reasons for such a low rejection ratio.

\begin{tabular}{|c|c|c|}
\hline Amplitude distribution & Theoretical rejection ratio & Measured rejection ratio \\
\hline $1: 1: 1$ & 275 & 188 \\
\hline $1: 2: 1$ & 3100 & 42 \\
\hline
\end{tabular}

Table 1. Theoretical and measured rejection ratios in the case of a three-beam nulling interferometer. The spectral band goes from 500 to $650 \mathrm{~nm}$.

\section{SPECTRAL MEASUREMENTS}

In order to achieve a rejection ratio of a few thousand, the intensity of the different beams should be matched with an accuracy of the order of $1 \%$. In a monochromatic set-up, this is easily achieved using amplitude-matching devices such as a knife-edge, a diaphragm, ... In a wide spectral band, this condition must be fulfilled for each wavelength, implying that each component must be achromatic or identical for each beam.

Some spectral mismatchings have already been pointed out in an earlier paper ${ }^{6}$ but, because of low light levels, only indirect measurements of the spectra by Fourier analysis of pairwise interferograms were possible. To confirm these spectral mismatchings, we performed direct measurements, using two different methods.

At first, we compared the intensity of the different beams at certain wavelengths, using narrow-band interference filters (10 nm FWHM at the central wavelengths 502, 551, 576, 594 and $643 \mathrm{~nm}$ ). In order to see whether the single-mode fiber affected the spectrum, measurements have been performed both before and after the fiber. The results are shown in Fig. 2(b). Each point represents the relative intensity of beams $B 1$ and $B 2$ with respect to the third beam (B3). In an ideally achromatic set-up, there would not be any spectral mismatching and these relative spectra would be constant.

We then used a multi-mode fiber spectrometer to directly measure the whole spectra. Two series of measurements of the three beams are depicted in Fig. 2(a). Note that we applied some numerical filtering to smoothen the spectra. We can see that, for each beam, the two series of measurements look very different. Indeed, the fiber was not very stable and the spectra were strongly depending on the alignment or the bending of the fiber. Therefore, these absolute measurements as such were not reliable. Nevertheless, as long as we keep the same 


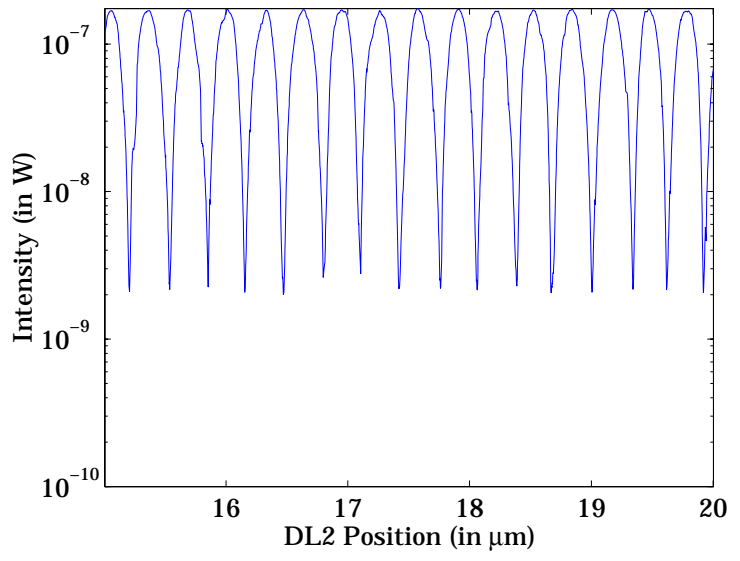

(a)

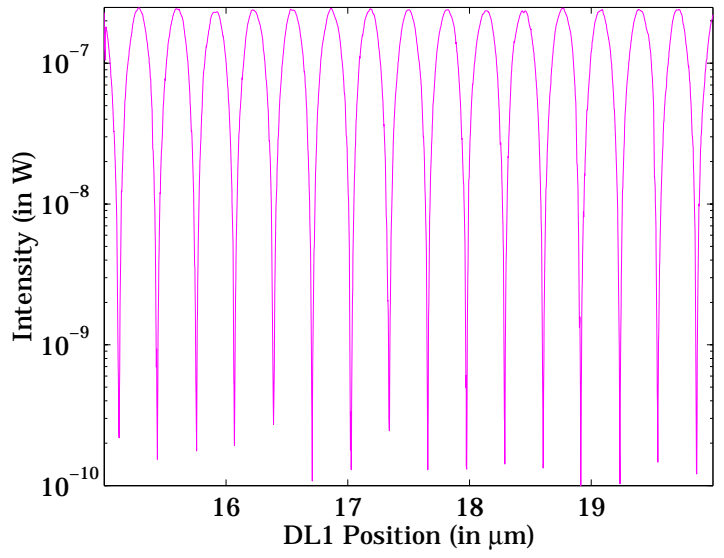

(b)

Figure 3. Interference patterns with laser light (He-Ne) without polarizer between (a) beams 1 and $3(R=87)$ and (b) beams 2 and $3(R=2770)$.

configuration for all the beams, we can compare the spectra in a more reliable way. Indeed, if we compare these two series of spectra with the spectrum of the third beam, (see Fig. 2(b)), we can see that the relative measurements are quite repeatable while the absolute spectra were totally different. Furthermore, we can see that they match quite nicely the first measurements performed with the interference filters, confirming the reliability of both methods. All measurements show large spectral mismatchings (see Fig. 2(b)), mainly between the beams 2 and 3 (more than $20 \%$ mismatching).

As already mentioned previously, amplitude-matching in a wide spectral band is not an easy task, since the amplitude-matching method can also affect the phase and the shape of the spectrum. Indeed, some measurements of the spectra with a knife-edge or a diaphragm showed that these devices affect the shape of the spectrum, but the measurements depicted in Fig. 2(b) already show unacceptable mismatchings without any amplitude-matching device. Another possible reason for spectral mismatching would be different wavelength-dependent coupling efficiency with the single-mode optical fiber. ${ }^{9}$ Different coupling efficiencies could be caused by misalignments or aberrations, but we can see on Fig. 2(b) that the influence of the fiber is not so important. Indeed, measurements have been performed before and after the fiber and they do not show significant differences. Another possible reason would be a difference in the coatings of the beam-splitters. Indeed, by swapping two of the beam-splitters, we found similar results but this time with the most important spectral mismatching between the first and the third beams. From this measurement, we conclude that the spectral mismatching is thus mainly caused by coating imperfections of the beam-splitters.

To correct for this mismatching, we should either replace the beam-splitters or find a way to chromatically change the amplitudes. The latter might be an interesting option since, as shown in an earlier paper, ${ }^{10}$ a much higher rejection ratio (theoretically infinite) could be reached.

\section{POLARIZATION MEASUREMENTS}

In order to check the alignment of the set-up, we perform two-beam interferometry with a He-Ne laser (see Fig. 3 ). We noticed that the rejection ratio between beams 2 and 3 was much higher than between beams 1 and 3 (see Table 2). In a monochromatic set-up, amplitude-matching is trivial. The phase-matching is also nicely performed by changing the position of the delay line with 1-nm steps. In the monochromatic case, the only remaining possibility for such an important difference is polarization mismatching. This problem should then be solved (in the monochromatic case) by placing a linear polarizer (Glan-Laser polarizer) after recombination before the single-mode optical fiber. Measurements with and without polarizer are summarized in Table 2 . We can see that the rejection ratios are of the same order when we use a linear polarizer after recombination. 


\begin{tabular}{|c|c|c|c|}
\hline & Beams 1 and 2 & Beams 1 and 3 & Beams 2 and 3 \\
\hline Without polarizers & 94 & 87 & 2770 \\
\hline GL-polarizer after recombination & 15000 & 5140 & 5710 \\
\hline GL-polarizers after recombination and before splitting & 6390 & 18000 & $/$ \\
\hline
\end{tabular}

Table 2. Measured rejection ratios in the case of a two-beam monochromatic nulling interferometer.

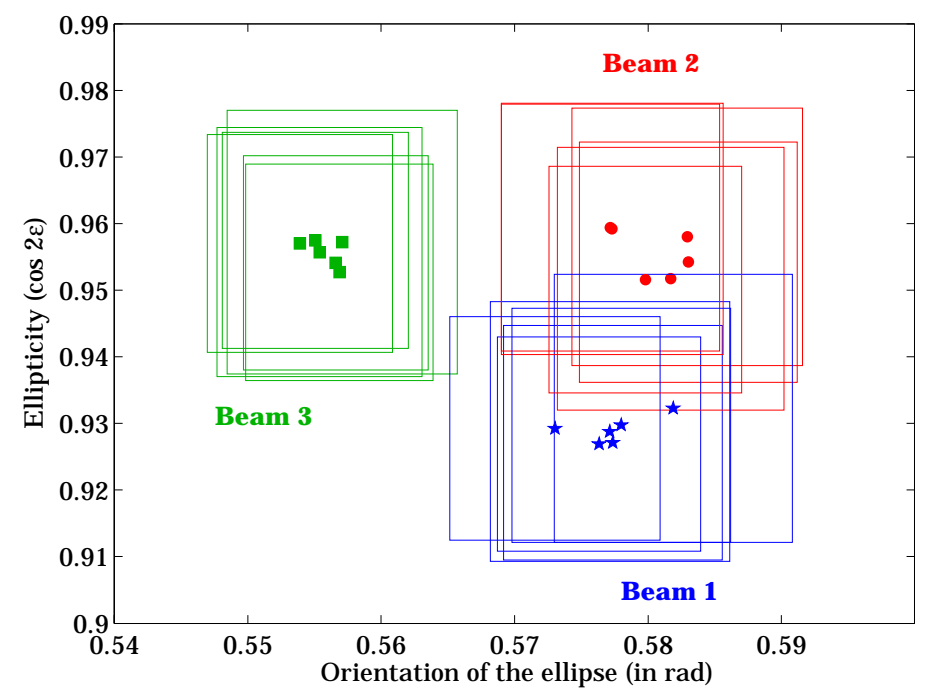

Figure 4. Polarization of the three beams with laser light (He-Ne) represented by their ellipticity and long-axis orientation. Each point represents one measurement in the ellipticity-orientation space and the rectangles represent the uncertainty of these measurements

In order to quantify these polarization mismatchings, we directly measured the polarization of the three beams with the He-Ne laser (before the single-mode fiber). For each beam, we measured the intensity after a linear polarizer oriented at $0^{\circ}\left(I_{0}\right), 45^{\circ}\left(I_{45}\right), 90^{\circ}\left(I_{90}\right)$ and $135^{\circ}\left(I_{135}\right)$. From these intensities, we can calculate three of the Stokes parameters using

$$
\begin{gathered}
S_{0}=I_{0}+I_{90}, \\
S_{1}=I_{0}-I_{90}, \\
S_{2}=I_{45}-I_{135} .
\end{gathered}
$$

With the general state of polarization being an ellipse, we can qualify the polarization of each beam by an ellipticity $\epsilon$ and an angle $\alpha$ giving the orientation of the ellipse (and a sense). The orientation $\alpha$ and the ellipticity $\epsilon$ can be calculated from the Stokes parameters using

$$
\begin{gathered}
\alpha=1 / 2 \arctan S_{2} / S_{1} \\
\epsilon=1 / 2 \arccos \sqrt{\frac{S_{1}^{2}+S_{2}{ }^{2}}{S_{0}}} .
\end{gathered}
$$

The polarization measurements are depicted in Fig. 4. Each point represents a measurement in the ellipticityorientation domain and the rectangle around that point represents the uncertainty of the measurement. Even though the uncertainties were relatively important, we can clearly see three different clusters of points, each of them representing one beam. These measurements confirm the presence of polarization mismatchings between the beams. The reason for these polarization mismatchings is probably some birefringence induced in the beamsplitters. Note that these measurements have only been performed for one wavelength. Therefore, it does not give any qualitative information for the whole spectral band. Nevertheless, it allows us to think that similar mismatchings are present in the whole spectral band. 


\section{LATEST RESULTS}

In Section 4, we showed that there are spectral mismatchings between the beams in our set-up. The best way to solve this mismatching problem would have been to replace the beam-splitters. However, we chose to limit the spectral band using color filters to a region where mismatching was less problematic (from 550 to $700 \mathrm{~nm}$ ).

The polarization mismatching reported in Section 5 is also not trivial to solve. Using a linear polarizer after recombination provides quite good results with laser light. Unfortunately, in a wide spectral band, the induced birefringence is probably wavelength-dependent due to dispersion. Polarization mismatching will be taken care of by the polarizer but a different birefringence will give rise to a different dispersion, that cannot be easily compensated.

All the results, with laser or white light, with or without polarizers, are summarized in Table 3. We can see that, with the laser, the two- or three-beam rejection ratio is $10^{4}$, which is probably the maximal reachable with our set-up due to vibrations. The white-light measurements show no significant difference with or without polarizers. Therefore, the polarization mismatching is probably not the limiting factor. Two of the white-light

\begin{tabular}{|c|c|c|c|c|}
\hline & \multicolumn{3}{|c|}{ Two beams } & Three beams \\
\hline & Beams 1 and 2 & Beams 1 and 3 & Beams 2 and 3 & Beams 1,2 and 3 \\
\hline \multicolumn{5}{|l|}{ Laser } \\
\hline No polarizer & 94 & 87 & 2770 & 1 \\
\hline One polarizer & 15000 & 5140 & 5710 & 11027 \\
\hline Two polarizers & 6390 & 18000 & 1 & 1 \\
\hline \multicolumn{5}{|l|}{ White light } \\
\hline No polarizer & $T$ & 40.8 & 46.8 & 101.7 \\
\hline One polarizer & 1 & 107.7 & 74.2 & 82.2 \\
\hline Two polarizers & 1 & 73.3 & 54.8 & 97.4 \\
\hline
\end{tabular}

Table 3. Measured rejection ratios in the case of a two-beam and a three-beam nulling interferometer, with laser and white light. Measurements have been taken without polarizer, with one polarizer after recombination or with one polarizer after recombination and one before splitting.

measurements are depicted in Fig. 5. Fig. 5(a) is the measured interference pattern between beams 1 and 3, with one polarizer before splitting and one after recombination and Fig. 5(b) is the interference pattern of the three beams without polarizer. In the ideal case, the two-beam fringes should be symmetric with respect to the maximum. We can clearly see in Fig. 5(a) that it is not the case. We can also see in Fig. 5(b) that the minimum and the maximum of the interference pattern have the same ordinate. In terms of phase-shifts with respect to the maximum, we can say that the minimum is located at $(\pi, 0)$ while we can show ${ }^{7}$ that it should always be at $(\pi, 2 \pi)$ (not depicted here), no matter what the amplitude distribution or the spectral mismatching (See Fig. 6).

One phenomenon that could explain both asymmetries or "defects" in the interference patterns is dispersion. Indeed, let us consider the interference between two beams. If one of the beams has a longer path in glass, it will give rise to an asymmetric pattern. The "level" of asymmetry depends on the path difference in glass. In the three-beam case, different paths in glass could also give similar patterns to the one measured.

A configuration where the minimum is at $(\pi, 0)$ always gives a low rejection ratio compared to the $(\pi, 2 \pi)$ configuration. In this case, it might be worthwhile to compensate for these path differences. On the other hand, we can show that there are optimal path differences for which the rejection ratio is much higher than without any path difference (see Table 4). Depending on the goal we want to reach, we might either compensate or optimize these differences. Indeed, in order to demonstrate the pure delay-line approach, they have to be compensated. However, the highest rejection ratio will be reached by optimizing the path differences.

\section{CONCLUSIONS}

We performed a quantitative analysis of a three-beam nulling interferometer using delay lines only. 


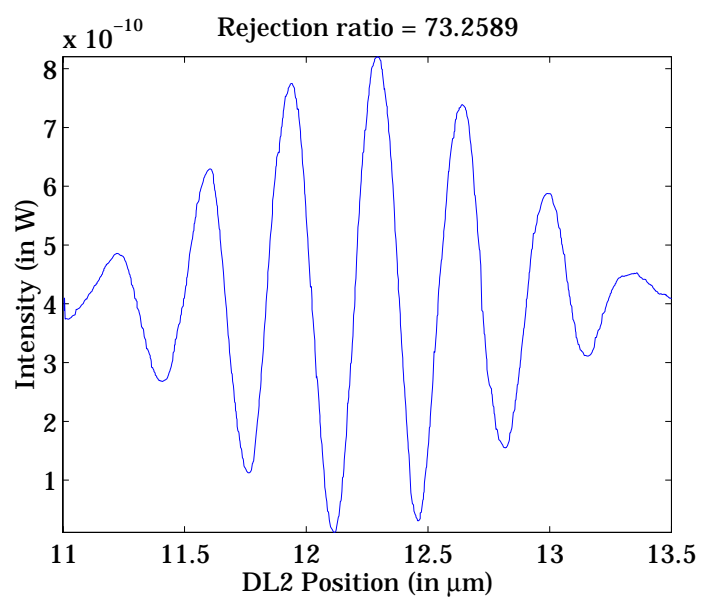

(a)

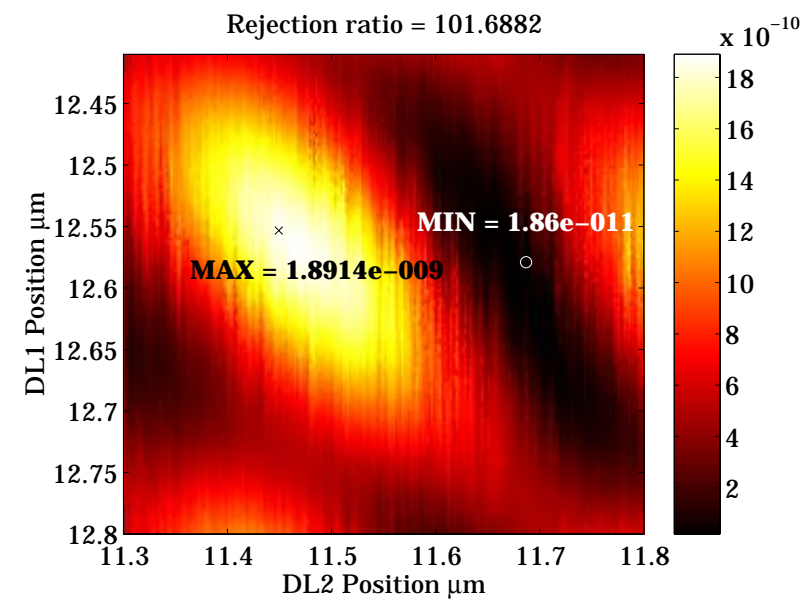

(b)

Figure 5. Interference patterns with white light (a) with two beams $(R=73.3)$ and (b) three beams $(R=101.7)$.

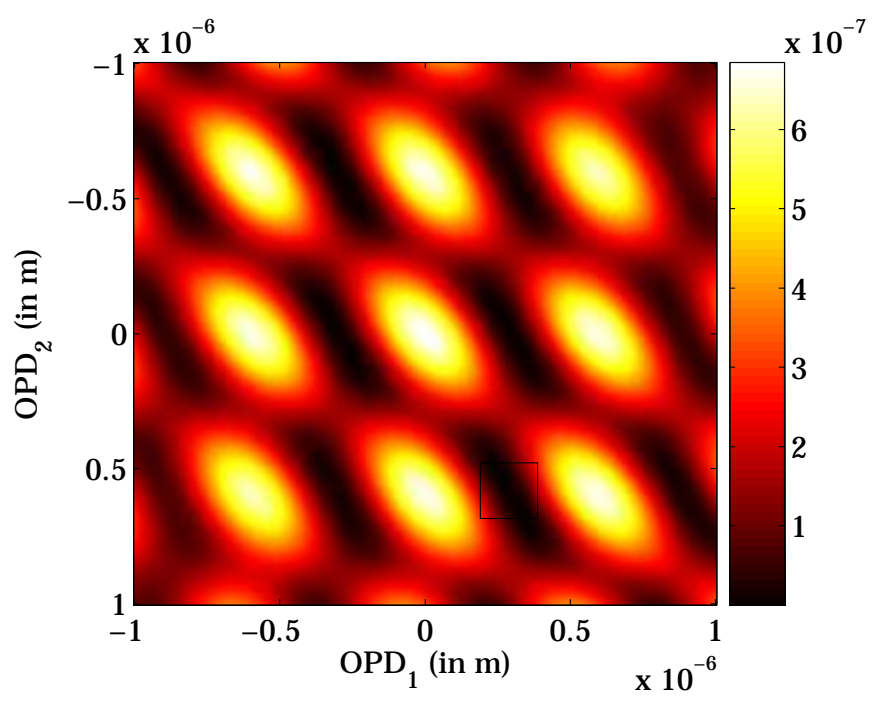

Figure 6. Theoretical white-light interference pattern. The maximum is at $(0,0)$ and the minimum, indicated by the black rectangle, is at $(\pi, 2 \pi)$.

\begin{tabular}{|c|c|c|}
\hline & Without path-differences in BK7 & With optimized path differences in BK7 \\
\hline Two beams & 150 & 2000 \\
\hline Three beams & 3000 & $9.10^{5}$ \\
\hline
\end{tabular}

Table 4. Theoretical rejection ratios with or without path-differences in BK7. 
We have measured, in a direct way, the spectra of the three beams in two different ways: with narrow-band interference filters and with a fiber spectrometer. We have shown that, even though the methods were not very reliable for absolute measurements, we obtained very similar and repeatable results for both methods. With these results, we have pointed out some important spectral mismatching (of the order of 20\%), mainly due to imperfections in the coating of the beam-splitters.

We have also quantified polarization mismatchings between the beams in the monochromatic case. We have seen that both ellipticity and orientation differ from one beam to the other, drastically limiting the rejection ratio in the monochromatic case. We have seen that it was not trivial to fix that problem in a wide spectral band. The best way to solve both polarization and spectral mismatchings is probably to replace the beam-splitters.

We have also measured asymmetries in the interference patterns, probably caused by different path lengths in glass. These asymmetries should either be compensated or optimized. Indeed, in order to demonstrate the "delay-line principle", these path differences should be corrected. We could also use and optimize this dispersion effect to fundamentally improve the performance of a nulling interferometer.

\section{ACKNOWLEDGMENTS}

We acknowledge the help of François Houndonougbo for performing some of the measurements and Amir Vosteen for some fruitful discussion on technical aspects of the experiments.

This research was supported by the Knowledge center for Aperture Synthesis, a collaboration of TNO and Delft University of Technology, The Netherlands.

\section{REFERENCES}

1. M. Mayor and D. Queloz, "A jupiter-mass companion to a solar-type star," Nature 378, pp. 355-359, 1995.

2. N. Woolf and J. R. Angel, "Astronomical searches for earth-like planets and signs of life," Astron. Astrophys. 36, pp. 507-537, 1998.

3. G. W. Marcy and R. P. Butler, "Detection of extrasolar giant planets," Astron. Astrophys. 36, pp. 57-97, 1998.

4. R. Bracewell, "Detecting nonsolar planets by spinning infrared interferometer," Nature 274, pp. 780-781, 1978.

5. A. L. Mieremet and J. J. M. Braat, "Nulling interferometry without achromatic phase shifters," Appl. Opt. 41, pp. 4697-4704, 2002.

6. C. van der Avoort, A. L. Mieremet, S. F. Pereira, and J. J. M. Braat, "Demonstration of nulling using delay line phase shifters," Proc. SPIE 5491, pp. 808-815, 2004.

7. A. L. Mieremet and J. J. M. Braat, "Deep nulling by means of multiple-beam recombination," Appl. Opt. 42, pp. 1867-1875, 2003.

8. B. Mennesson, M. Ollivier, and C. Ruilier, "Use of single-mode waveguides to correct the optical defects of a nulling interferometer," J. Opt. Soc. Am. A 19, pp. 596-602, 2002.

9. J. Spronck, S. F. Pereira, and J. J. M. Braat, "Chromatic coupling efficiency of a single-mode optical fiber for nulling interferometry," Proc. Symposium IEEE/LEOS Benelux Chapter, pp. 277-280, 2005.

10. J. Spronck, S. F. Pereira, and J. J. M. Braat, "Chromatism compensation in wide-band nulling interferometry for exoplanet detection," Appl. Opt. 45, pp. 597-604, 2006. 